\title{
Estudio de volumen molar y refracción molar de miscelas de triglicéridos (triacetina, tributirina o tricaprilina) y alcoholes (etanol, 1-butanol o 1-hexanol)
}

\author{
Por M. Rodríguez Rodríguez, M. Galán Vallejo y M.a J. Muñoz Cueto \\ Departamento de Ingeniería Química. Universidad de Cádiz. \\ Apdo. n. 0. Puerto Real. 11510 - Cádiz. España.
}

\section{RESUMEN}

Estudio de volumen molar y refracción molar de miscelas de triglicéridos (triacetina, tributirina o tricaprilina) y alcoholes (etanol, 1-butanol o 1-hexanol)

Los valores de la refracción molar en todas las miscelas estudiadas presentan variaciones lineales frente a la fracción molar de triglicérido, la misma variación que presentan los valores del volumen molar de las miscelas tributirina y tricaprilina en butanol. Sin embargo, en las miscelas de tributirina-etanol se encuentran contracciones de volumen mientras que en las de triacetina-butanol y tributirina-hexanol expansiones, atribuidas en ambos casos a la forma de estructurarse las moléculas de alcohol y triglicérido en las miscelas.

Por otra parte, se encuentra una variación lineal entre el volumen molar de las miscelas y la temperatura, y se establece una relación lineal entre el coeficiente de dilatación térmico molar a presión constante y la fracción molar de triglicérido en los cinco sistemas estudiados.

PALABRAS-CLAVE: Alcohol - Miscela - Refracción molar - Triglicérido - Volumen molar.

\section{SUMMARY}

Study of molar volume and refractioin of triglyceride (triacetin, tributyrin or tricaprylin) -alcohol (ethanol, 1-butanol or 1-hexanol) mixtures.

Values of molar refraction for every studied mixtures show linear plots versus molar fractions of triglyceride. Values of molar volume show this behaviour, but only for tributyrin-butanol or tricaprylin-butanol miscellas. However, in tributyrin-ethanol mixtures, volume contractions have been found, whereas triacetin-butanol and tributyrin-hexanol show volume expansions. These facts are related to the mode of being structured of the alcohol and triglyceride molecules in mixtures.

A linear relationship between molar volume and temperature have been found, and also a linear dependence between the coefficient of thermal expansion at constant pressure and the molar fraction of triglyceride.

KEY-WORDS: Alcohol - Miscella - Molar refraction - Molar volume Triglyceride.

\section{INTRODUCCION}

En comunicaciones de la serie titulada "Estudios físico-químicos de miscelas de aceites vegetales", se ha estudiado el comportamiento de las miscelas de los ácidos láurico y oleico (Flores, 1974; Flores, 1977a, b, c:
Flores, 1979a, b); de los ésteres de metilo y $n$-hexilo del ácido láurico (Flores, 1983; Flores, 1984); de aceites de soja o girasol (Flores, 1983; Flores, 1987) y de los ésteres metílicos de los ácidos mirístico, palmítico y esteárico (Muñoz, 1990a, b; Muñoz, 1991), en diferentes disolventes.

En el presente trabajo se aborda el estudio de las miscelas de triglicéridos (triacetina, tributirina o tricaprilina) y alcoholes (etanol, 1-butanol o 1-hexanol) con objeto de establecer el efecto que ejerce la longitud de la cadena carbonada del alcohol así como el tamaño del triglicérido sobre el valor de diferentes propiedades.

En posteriores comunicaciones se abordará el estudio de la viscosidad de las mencionadas miscelas.

\section{PARTE EXPERIMENTAL}

\subsection{Productos empleados}

Los productos estudiados, según la firma suministradora, presentan las características que se indican a continuación:

\begin{tabular}{lcc}
\hline & $\begin{array}{c}\text { Pureza } \\
\text { (cromatografia gaseosa) }\end{array}$ & $\begin{array}{c}\text { Peso molecular } \\
\text { medio }\end{array}$ \\
\hline Etanol & $>99,8 \%$ & 46,07 \\
1-butanol & $>99,5 \%$ & 74,12 \\
1-hexanol & $>98,0 \%$ & 102,18 \\
triacetina & $>99,0 \%$ & 218,21 \\
tributirina & $>98,0 \%$ & 302,37 \\
tricaprilina & $>97,0 \%$ & 470,70 \\
\hline
\end{tabular}

\subsection{Preparación de las miscelas}

La preparación de las miscelas se ha realizado por pesada, determinándose la densidad e índice de refracción de aquellas que se presentan como monofásica líquida a la temperatura de estudio. La balanza utilizada 
para la pesada es de tipo monoplato Mettler P1210, capaz de apreciar centésimas de gramo.

\subsection{Técnicas experimentales}

Se han determinado los valores de densidad e índice de refracción correspondientes a las miscelas preparadas, entre 278 y $313 \mathrm{~K}$, a intervalos de $5 \mathrm{~K}$.

Para termostatar las miscelas se ha utilizado un baño Hetofrig, que permite controlar la temperatura en el intervalo estudiado con una precisión de $\pm 0,1 \mathrm{~K}$.

Los valores de densidad se han determinado mediante un densímetro digital DMA 46 con termostato incorporado.

Los valores del índice de refracción se han determinado utilizando un refractómetro tipo Abbé de la firma ATAGO cuya amplitud de escala es de 1,3000 a 1,7100.

\subsection{Resultados experimentales}

Los valores experimentales obtenidos se recogen en las Tablas I a V, correspondiendo a cada fracción molar y temperatura de arriba a abajo, densidad (gramos/centímetro cúbico) e índice de refracción.

El último dígito que aparece en cada una de las medidas es aquel que se ve afectado por los límites de confianza del método experimental.

\section{DISCUSION DE RESULTADOS}

\subsection{Volumen molar de las miscelas}

El valor del volumen molar experimental de las miscelas (centímetros cúbicos $/ \mathrm{mol}$ ) se obtiene del cociente entre los correspondientes valores del peso molecular medio y de la densidad (gramos/centímetros cúbico).

\section{Relación volumen molar-composición}

Para cada tipo de miscela y temperatura se encuentra una relación lineal entre el volumen molar experimental de la miscela y la fracción molar del triglicérido que puede expresarse de la forma:

$$
V=\left(\bar{V}_{2}-\bar{V}_{1}\right) x+\bar{V}_{1}
$$

donde " $V$ " es el valor de volumen molar de la miscela, " $x$ " es la fracción molar de triglicérido en la miscela y " $\bar{V}_{2}$ " y " $\bar{V}$ " son parámetros de la recta de regresión, que deben corresponder respectivamente a los valores de los volúmenes molares del triglicérido y del alcohol que forman la miscela, admitiendo la hipótesis de que ésta se comporta como mezcla ideal de dos líquidos orgánicos sin ionizar.

La regresión lineal encontrada presenta en todos los casos un valor del "porcentaje de varianza no justificada" $\left(V N J=100\left(1-R^{2}\right)(N-1) /(N-2)\right.$, donde " $R$ " es el coeficiente de regresión lineal y " $N$ " es el número de pares de datos utilizados para calcularlo) inferior a 0,0023.

Tabla 1

Miscelas de triacetina-butanol, valores de densidad $\left(\mathrm{g} . \mathrm{cm}^{-3}\right)$ e indices de refracción

\begin{tabular}{|c|c|c|c|c|c|c|c|c|}
\hline f.m. & $278 \mathrm{~K}$ & $283 \mathrm{~K}$ & $288 \mathrm{~K}$ & $293 \mathrm{~K}$ & $298 \mathrm{~K}$ & $303 \mathrm{~K}$ & $308 \mathrm{~K}$ & $313 \mathrm{~K}$ \\
\hline 0,0 & 0,8218 & $\begin{array}{l}0,8178 \\
1,4020\end{array}$ & $\begin{array}{l}0,8138 \\
1,4005\end{array}$ & $\begin{array}{l}0,8100 \\
1,3990\end{array}$ & $\begin{array}{l}0,8061 \\
1,3970\end{array}$ & $\begin{array}{l}0,8021 \\
1,3950\end{array}$ & $\begin{array}{l}0,7983 \\
1,3930\end{array}$ & $\begin{array}{l}0,7943 \\
1,3910\end{array}$ \\
\hline 0,1 & $\begin{array}{c}0,8867 \\
-\end{array}$ & $\begin{array}{l}0,8825 \\
1,4075\end{array}$ & $\begin{array}{l}0,8782 \\
1,4055\end{array}$ & $\begin{array}{l}0,8737 \\
1,4030\end{array}$ & $\begin{array}{l}0,8697 \\
1,4020\end{array}$ & $\begin{array}{l}0,8654 \\
1,4005\end{array}$ & $\begin{array}{l}0,8611 \\
1,3980\end{array}$ & $\begin{array}{l}0,8569 \\
1,3965 \\
\end{array}$ \\
\hline 0,2 & 0,9404 & $\begin{array}{l}0,9358 \\
1,4120\end{array}$ & $\begin{array}{l}0,9312 \\
1,4110\end{array}$ & $\begin{array}{l}0,9265 \\
1,4080\end{array}$ & $\begin{array}{l}0,9222 \\
1,4060\end{array}$ & $\begin{array}{l}0,9176 \\
1,4045\end{array}$ & $\begin{array}{l}0,9130 \\
1,4030\end{array}$ & $\begin{array}{l}0,9085 \\
1,4010\end{array}$ \\
\hline 0,3 & $\begin{array}{c}0,9856 \\
-\end{array}$ & $\begin{array}{l}0,9808 \\
1,4160\end{array}$ & $\begin{array}{l}0,9761 \\
1,4140\end{array}$ & $\begin{array}{l}0,9709 \\
1,4120\end{array}$ & $\begin{array}{l}0,9664 \\
1,4105\end{array}$ & $\begin{array}{l}0,9616 \\
1,4085\end{array}$ & $\begin{array}{l}0,9568 \\
1,4065\end{array}$ & $\begin{array}{l}0,9521 \\
1,4050\end{array}$ \\
\hline 0,4 & $\begin{array}{c}1,0246 \\
-\end{array}$ & $\begin{array}{l}1,0196 \\
1,4200\end{array}$ & $\begin{array}{l}1,0147 \\
1,4185\end{array}$ & $\begin{array}{l}1,0098 \\
1,4175\end{array}$ & $\begin{array}{l}1,0049 \\
1,4145\end{array}$ & $\begin{array}{l}1,0002 \\
1,4120\end{array}$ & $\begin{array}{l}0,9948 \\
1,4100\end{array}$ & $\begin{array}{l}0,9901 \\
1,4080\end{array}$ \\
\hline 0,5 & $\begin{array}{c}1,0580 \\
-\end{array}$ & $\begin{array}{l}1,0530 \\
1,4230\end{array}$ & $\begin{array}{l}1,0479 \\
1,4210\end{array}$ & $\begin{array}{l}1,0425 \\
1,4195\end{array}$ & $\begin{array}{l}1,0378 \\
1,4175\end{array}$ & $\begin{array}{l}1,0331 \\
1,4160\end{array}$ & $\begin{array}{l}1,0276 \\
1,4135\end{array}$ & $\begin{array}{l}1,0227 \\
1,4110\end{array}$ \\
\hline 0,6 & $\begin{array}{c}1,0872 \\
-\end{array}$ & $\begin{array}{l}1,0820 \\
1,4260\end{array}$ & $\begin{array}{l}1,0768 \\
1,4240\end{array}$ & $\begin{array}{l}1,0713 \\
1,4220\end{array}$ & $\begin{array}{l}1,0666 \\
1,4200\end{array}$ & $\begin{array}{l}1,0616 \\
1,4180\end{array}$ & $\begin{array}{l}1,0561 \\
1,4160\end{array}$ & $\begin{array}{l}1,0509 \\
1,4140\end{array}$ \\
\hline 0,7 & $\begin{array}{c}1,1128 \\
-\end{array}$ & $\begin{array}{l}1,1075 \\
1,4280\end{array}$ & $\begin{array}{l}1,1023 \\
1,4260\end{array}$ & $\begin{array}{l}1,0968 \\
1,4240\end{array}$ & $\begin{array}{l}1,0918 \\
1,4225\end{array}$ & $\begin{array}{l}1,0866 \\
1,4200\end{array}$ & $\begin{array}{l}1,0813 \\
1,4180\end{array}$ & $\begin{array}{l}1,0760 \\
1,4165\end{array}$ \\
\hline 0,8 & $\begin{array}{c}1,1360 \\
-\end{array}$ & $\begin{array}{l}1,1306 \\
1,4300\end{array}$ & $\begin{array}{l}1,1252 \\
1,4285\end{array}$ & $\begin{array}{l}1,1196 \\
1,4265\end{array}$ & $\begin{array}{l}1,1146 \\
1,4250\end{array}$ & $\begin{array}{l}1,1091 \\
1,4230\end{array}$ & $\begin{array}{l}1,1036 \\
1,4210\end{array}$ & $\begin{array}{l}1,0982 \\
1,4190\end{array}$ \\
\hline 0,9 & $\begin{array}{c}1,1565 \\
-\end{array}$ & $\begin{array}{l}1,1512 \\
1,4320\end{array}$ & $\begin{array}{l}1,1455 \\
1,4305\end{array}$ & $\begin{array}{l}1,1404 \\
1,4285\end{array}$ & $\begin{array}{l}1,1353 \\
1,4265\end{array}$ & $\begin{array}{l}1,1299 \\
1,4245\end{array}$ & $\begin{array}{l}1,1244 \\
1,4225\end{array}$ & $\begin{array}{l}1,1191 \\
1,4210\end{array}$ \\
\hline 1,0 & $\begin{array}{c}1,1755 \\
-\end{array}$ & $\begin{array}{l}1,1698 \\
1,4345\end{array}$ & $\begin{array}{l}1,1642 \\
1,4325\end{array}$ & $\begin{array}{l}1,1586 \\
1,4305\end{array}$ & $\begin{array}{l}1,1529 \\
1,4285\end{array}$ & $\begin{array}{l}1,1472 \\
1,4265\end{array}$ & $\begin{array}{l}1,1415 \\
1,4245\end{array}$ & $\begin{array}{l}1,1358 \\
1,4225\end{array}$ \\
\hline
\end{tabular}


Tabla II

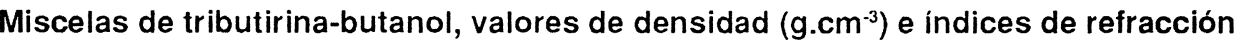

\begin{tabular}{ccccccccc}
\hline f.m. & $\mathbf{2 7 8} \mathbf{K}$ & $\mathbf{2 8 3} \mathbf{K}$ & $\mathbf{2 8 8} \mathbf{K}$ & $\mathbf{2 9 3} \mathbf{K}$ & $\mathbf{2 9 8} \mathbf{K}$ & $\mathbf{3 0 3} \mathbf{K}$ & $\mathbf{3 0 8} \mathbf{K}$ & $\mathbf{3 1 3} \mathbf{K}$ \\
\hline \multirow{2}{*}{0,0} & $\mathbf{2}, 8218$ & 0,8178 & 0,8138 & 0,8100 & 0,8061 & 0,8021 & 0,7983 & 0,7943 \\
& - & 1,4020 & 1,4005 & 1,3990 & 1,3970 & 1,3950 & 1,3930 & 1,3910 \\
\hline 0,1 & 0,8807 & 0,8765 & 0,8723 & 0,8680 & 0,8640 & 0,8599 & 0,8557 & 0,8515 \\
& - & 1,4110 & 1,4090 & 1,4080 & 1,4050 & 1,4040 & 1,4020 & 1,4000 \\
\hline 0,2 & 0,9215 & 0,9172 & 0,9127 & 0,9084 & 0,9042 & 0,8998 & 0,8957 & 0,8911 \\
& - & 1,4180 & 1,4160 & 1,4150 & 1,4130 & 1,4110 & 1,4090 & 1,4080 \\
\hline 0,3 & 0,9518 & 0,9474 & 0,9429 & 0,9382 & 0,9339 & 0,9294 & 0,9251 & 0,9205 \\
& - & 1,4230 & 1,4210 & 1,4190 & 1,4170 & 1,4160 & 1,4130 & 1,4120 \\
\hline 0,4 & 0,9748 & 0,9703 & 0,9657 & 0,9611 & 0,9566 & 0,9521 & 0,9478 & 0,9431 \\
& - & 1,4265 & 1,4250 & 1,4230 & 1,4210 & 1,4195 & 1,4180 & 1,4165 \\
\hline 0,5 & 0,9928 & 0,9882 & 0,9836 & 0,9790 & 0,9744 & 0,9699 & 0,9654 & 0,9607 \\
& - & 1,4300 & 1,4280 & 1,4260 & 1,4240 & 1,4230 & 1,4200 & 1,4185 \\
\hline 0,6 & 1,0077 & 1,0031 & 0,9985 & 0,9938 & 0,9893 & 0,9847 & 0,9801 & 0,9754 \\
& - & 1,4320 & 1,4300 & 1,4280 & 1,4270 & 1,4245 & 1,4230 & 1,4210 \\
\hline 0,7 & 1,0200 & 1,0153 & 1,0106 & 1,0060 & 1,0014 & 0,9967 & 0,9922 & 0,9874 \\
& - & 1,4340 & 1,4320 & 1,4300 & 1,4280 & 1,4260 & 1,4245 & 1,4225 \\
\hline 0,8 & 1,0300 & 1,0254 & 1,0207 & 1,0160 & 1,0115 & 1,0068 & 1,0022 & 0,9975 \\
& - & 1,4360 & 1,4340 & 1,4320 & 1,4300 & 1,4280 & 1,4260 & 1,4240 \\
\hline 0,9 & 1,0391 & 1,0344 & 1,0297 & 1,0251 & 1,0205 & 1,0157 & 1,0112 & 1,0064 \\
& - & 1,4370 & 1,4355 & 1,4335 & 1,4315 & 1,4300 & 1,4280 & 1,4260 \\
\hline 1,0 & 1,0468 & 1,0421 & 1,0374 & 1,0327 & 1,0280 & 1,0233 & 1,0187 & 1,0139 \\
& - & 1,4385 & 1,4370 & 1,4350 & 1,4330 & 1,4310 & 1,4290 & 1,4270 \\
\hline & & & & & & &
\end{tabular}

Tabla III

Miscelas de tricaprilina-butanol, valores de densidad $\left(\mathrm{g} . \mathrm{cm}^{-3}\right)$ e índices de refracción

\begin{tabular}{ccccccccc}
\hline f.m. & $\mathbf{2 7 8} \mathbf{K}$ & $\mathbf{2 8 3} \mathbf{K}$ & $\mathbf{2 8 8} \mathbf{K}$ & $\mathbf{2 9 3} \mathbf{K}$ & $\mathbf{2 9 8} \mathbf{K}$ & $\mathbf{3 0 3} \mathbf{K}$ & $\mathbf{3 0 8} \mathbf{K}$ & $\mathbf{3 1 3} \mathbf{K}$ \\
\hline 0,0 & 0,8218 & 0,8178 & 0,8138 & 0,8100 & 0,8061 & 0,8021 & 0,7983 & 0,7943 \\
& - & 1,4020 & 1,4005 & 1,3990 & 1,3970 & 1,3950 & 1,3930 & 1,3910 \\
\hline 0,1 & 0,8760 & 0,8721 & 0,8680 & 0,8639 & 0,8604 & 0,8561 & 0,8523 & 0,8482 \\
& - & 1,4205 & 1,4190 & 1,4170 & 1,4150 & 1,4135 & 1,4120 & 1,4100 \\
\hline 0,2 & 0,9046 & 0,9006 & 0,8966 & 0,8925 & 0,8887 & 0,8846 & 0,8809 & 0,8767 \\
& - & 1,4300 & 1,4285 & 1,4265 & 1,4250 & 1,4230 & 1,4210 & 1,4190 \\
\hline 0,3 & 0,9225 & 0,9185 & 0,9145 & 0,9103 & 0,9067 & 0,9024 & 0,8987 & 0,8945 \\
& - & 1,4360 & 1,4345 & 1,4325 & 1,4310 & 1,4290 & 1,4270 & 1,4250 \\
\hline 0,4 & 0,9347 & 0,9307 & 0,9267 & 0,9225 & 0,9188 & 0,9146 & 0,9108 & 0,9067 \\
& - & 1,4400 & 1,4385 & 1,4365 & 1,4350 & 1,4330 & 1,4310 & 1,4290 \\
\hline 0,5 & 0,9434 & 0,9394 & 0,9355 & 0,9313 & 0,9275 & 0,9235 & 0,9197 & 0,9156 \\
& - & 1,4430 & 1,4415 & 1,4395 & 1,4375 & 1,4360 & 1,4340 & 1,4320 \\
\hline 0,6 & 0,9501 & 0,9461 & 0,9421 & 0,9381 & 0,9342 & 0,9302 & 0,9264 & 0,9223 \\
& - & 1,4450 & 1,4435 & 1,4420 & 1,4400 & 1,4380 & 1,4360 & 1,4345 \\
\hline 0,7 & 0,9553 & 0,9513 & 0,9474 & 0,9433 & 0,9395 & 0,9354 & 0,9317 & 0,9276 \\
& - & 1,4470 & 1,4455 & 1,4435 & 1,4420 & 1,4400 & 1,4380 & 1,4360 \\
\hline 0,8 & 0,9597 & 0,9557 & 0,9518 & 0,9478 & 0,9439 & 0,9398 & 0,9361 & 0,9320 \\
& - & 1,4485 & 1,4470 & 1,4450 & 1,4430 & 1,4415 & 1,4395 & 1,4375 \\
\hline 0,9 & 0,9633 & 0,9593 & 0,9554 & 0,9514 & 0,9476 & 0,9434 & 0,9397 & 0,9356 \\
& - & 1,4495 & 1,4480 & 1,4460 & 1,4445 & 1,4425 & 1,4410 & 1,4390 \\
\hline 1,0 & 0,9662 & 0,9623 & 0,9583 & 0,9544 & 0,9504 & 0,9464 & 0,9427 & 0,9386 \\
& - & 1,4505 & 1,4490 & 1,4470 & 1,4455 & 1,4435 & 1,4420 & 1,4400 \\
\hline & & & & & & & &
\end{tabular}


Tabla IV

Miscelas de tributirina-etanol, valores de densidad $\left(\mathrm{g} . \mathrm{cm}^{-3}\right)$ e indices de refracción

\begin{tabular}{ccccccccc}
\hline f.m. & $\mathbf{2 7 8} \mathbf{K}$ & $\mathbf{2 8 3} \mathbf{K}$ & $\mathbf{2 8 8} \mathbf{K}$ & $\mathbf{2 9 3} \mathbf{K}$ & $\mathbf{2 9 8} \mathbf{K}$ & $\mathbf{3 0 3} \mathbf{K}$ & $\mathbf{3 0 8} \mathbf{K}$ & $\mathbf{3 1 3} \mathbf{K}$ \\
\hline 0,0 & 0,8031 & 0,7986 & 0,7943 & 0,7898 & 0,7854 & 0,7808 & 0,7766 & 0,7721 \\
& - & 1,3650 & 1,3630 & 1,3610 & 1,3590 & 1,3570 & 1,3550 & 1,3530 \\
\hline 0,1 & 0,8921 & 0,8877 & 0,8835 & 0,8789 & 0,8742 & 0,8698 & 0,8657 & 0,8612 \\
& - & 1,3920 & 1,3900 & 1,3880 & 1,3865 & 1,3850 & 1,3830 & 1,3820 \\
\hline 0,2 & 0,9400 & 0,9356 & 0,9315 & 0,9268 & 0,9220 & 0,9178 & 0,9137 & 0,9091 \\
& - & 1,4070 & 1,4050 & 1,4030 & 1,4010 & 1,4000 & 1,3980 & 1,3960 \\
\hline 0,3 & 0,9708 & 0,9663 & 0,9620 & 0,9575 & 0,9525 & 0,9482 & 0,9440 & 0,9394 \\
& - & 1,4160 & 1,4140 & 1,4125 & 1,4110 & 1,4090 & 1,4075 & 1,4055 \\
\hline 0,4 & 0,9913 & 0,9868 & 0,9825 & 0,9778 & 0,9731 & 0,9684 & 0,9644 & 0,9597 \\
& - & 1,4225 & 1,4205 & 1,4190 & 1,4170 & 1,4160 & 1,4140 & 1,4120 \\
\hline 0,5 & 1,0066 & 1,0021 & 0,9978 & 0,9932 & 0,9884 & 0,9839 & 0,9797 & 0,9751 \\
& - & 1,4270 & 1,4250 & 1,4230 & 1,4220 & 1,4200 & 1,4185 & 1,4170 \\
\hline 0,6 & 1,0183 & 1,0138 & 1,0095 & 1,0047 & 0,9999 & 0,9952 & 0,9910 & 0,9864 \\
& - & 1,4305 & 1,4285 & 1,4270 & 1,4250 & 1,4240 & 1,4220 & 1,4200 \\
\hline 0,7 & 1,0277 & 1,0231 & 1,0187 & 1,0139 & 1,0091 & 1,0045 & 1,0002 & 0,9955 \\
& - & 1,4330 & 1,4310 & 1,4295 & 1,4275 & 1,4260 & 1,4240 & 1,4225 \\
\hline 0,8 & 1,0349 & 1,0302 & 1,0257 & 1,0209 & 1,0162 & 1,0115 & 1,0071 & 1,0024 \\
& - & 1,4355 & 1,4335 & 1,4315 & 1,4300 & 1,4280 & 1,4260 & 1,4240 \\
\hline 0,9 & 1,0413 & 1,0366 & 1,0320 & 1,0273 & 1,0226 & 1,0178 & 1,0134 & 1,0086 \\
& - & 1,4370 & 1,4350 & 1,4330 & 1,4315 & 1,4295 & 1,4275 & 1,4260 \\
\hline 1,0 & 1,0468 & 1,0421 & 1,0374 & 1,0327 & 1,0280 & 1,0233 & 1,0187 & 1,0139 \\
& - & 1,4385 & 1,4370 & 1,4350 & 1,4330 & 1,4310 & 1,4290 & 1,4270 \\
\hline & & & & & & &
\end{tabular}

En la tabla $V I$ se recogen los valores de los parámetros " $\bar{V}_{2}$ " y " $\bar{V}_{1}$ " correspondientes a cada una de las miscelas estudiadas, expresados en centímetros cúbi$\mathrm{cos} / \mathrm{mol}$, así como los valores experimentales de los volúmenes molares de los triglicéridos y alcoholes utilizados, cuyo error se estima en $\pm 0,1 \mathrm{~cm}^{3} \cdot \mathrm{mol}^{-1}$ para una probabilidad del $95 \%$. Los errores estimados en los valores de los parámetros de ajuste para una probabilidad del $95 \%$, expresados en centímetros cúbicos/mol son:

\begin{tabular}{lll}
\hline Miscela & $\overline{\boldsymbol{V}}_{2}$ & $\overline{\boldsymbol{V}}_{1}$ \\
\hline Triacetina-Butanol & $\pm 0,5$ & $\pm 0,2$ \\
Tributirina-Butanol & $\pm 0,2$ & $\pm 0,1$ \\
Tricaprilina-Butanol & $\pm 0,3$ & $\pm 0,1$ \\
Tributirina-Etanol & $\pm 0,3$ & $\pm 0,1$ \\
Tributirina-Hexanol & $\pm 0,4$ & $\pm 0,2$ \\
\hline
\end{tabular}

Del análisis de estos valores se puede establecer que el comportamiento de las miscelas tributirina y tricaprilina en butanol respecto al volumen molar, no difiere del correspondiente a la hipótesis de mezclas ideales anteriormente mencionada. En el caso de las miscelas tributirina-etanol; triacetina-butanol y tributirina-hexanol los valores del parámetro " $\bar{V}$," no comprende dentro de su margen de error a los valores experimentales del volumen molar correspondiente, si bien las desviaciones de mezcla ideal, para el nivel de probabilidad elegido, supone un error relativo inferior al $1 \%$.
Si se calculan los valores del volumen molar relativo en exceso, definido como:

$$
V_{R}^{E}=\frac{V_{\text {exp }}-V_{t}}{V_{\text {exp }}} \times 100
$$

siendo " $V_{\text {exp }}$ ", el volumen molar experimental de la miscela y " $V_{i}$ ", el volumen molar teórico de la miscela considerada como ideal, se observa cómo en el caso de las miscelas antes indicadas existen valores mayores que 0,20 (los valores inferiores se consideran dentro del error experimental) y en ningún caso superior a 0,3 que justifican las desviaciones de dichas miscelas respecto a la hipótesis de mezcla ideal. A modo de ilustración en la figura 1 se representan los valores del volumen molar relativo frente a la fracción molar de triglicérido a la temperatura de $303 \mathrm{~K}$.

En las miscelas tributirina-etanol, los valores de volumen molar relativo en exceso son negativos en el intervalo de tracción molar de triglicérido comprendido entre 0,1 y 0,7 lo que indica que existen contracciones de volumen. Estas se atribuyen a un aumento en el grado de estructuración de las miscelas debido a la existencia de fuertes interacciones entre las moléculas de los componentes, principalmente enlaces de hidrógeno y fuerzas intermoleculares, y al menor tamaño de las moléculas de etanol, ya que el aumento del número de átomos de carbono del alcohol origina impedimentos de tipo estérico que entorpecen la reordenación molecular de las miscelas (Jiménez, 1974). 
Tabla V

Miscelas de tributirina-hexanol, valores de densidad $\left(\mathrm{g} . \mathrm{cm}^{-3}\right)$ e índices de refracción

\begin{tabular}{ccccccccc}
\hline f.m. & $\mathbf{2 7 8 ~ K}$ & $\mathbf{2 8 3} \mathbf{K}$ & $\mathbf{2 8 8} \mathbf{K}$ & $\mathbf{2 9 3} \mathbf{K}$ & $\mathbf{2 9 8} \mathbf{K}$ & $\mathbf{3 0 3} \mathbf{K}$ & $\mathbf{3 0 8} \mathbf{K}$ & $\mathbf{3 1 3} \mathbf{K}$ \\
\hline 0,0 & 0,8298 & 0,8262 & 0,8226 & 0,8190 & 0,8150 & 0,8115 & 0,8079 & 0,8042 \\
& - & 1,4210 & 1,4190 & 1,4170 & 1,4155 & 1,4140 & 1,4120 & 1,4100 \\
\hline 0,1 & 0,8737 & 0,8698 & 0,8660 & 0,8619 & 0,8579 & 0,8541 & 0,8502 & 0,8463 \\
& - & 1,4240 & 1,4220 & 1,4200 & 1,4180 & 1,4170 & 1,4150 & 1,4130 \\
\hline 0,2 & 0,9085 & 0,9044 & 0,9005 & 0,8962 & 0,8921 & 0,8881 & 0,8840 & 0,8800 \\
& - & 1,4265 & 1,4250 & 1,4230 & 1,4210 & 1,4190 & 1,4170 & 1,4150 \\
\hline 0,3 & 0,9369 & 0,9327 & 0,9286 & 0,9242 & 0,9199 & 0,9157 & 0,9115 & 0,9073 \\
& - & 1,4290 & 1,4270 & 1,4255 & 1,4230 & 1,4215 & 1,4200 & 1,4175 \\
\hline 0,4 & 0,9606 & 0,9562 & 0,9520 & 0,9475 & 0,9430 & 0,9387 & 0,9344 & 0,9301 \\
& - & 1,4310 & 1,4290 & 1,4270 & 1,4250 & 1,4230 & 1,4215 & 1,4195 \\
\hline 0,5 & 0,9805 & 0,9760 & 0,9717 & 0,9672 & 0,9626 & 0,9582 & 0,9539 & 0,9494 \\
& - & 1,4320 & 1,4310 & 1,4290 & 1,4270 & 1,4250 & 1,4230 & 1,4210 \\
\hline 0,6 & 0,9975 & 0,9930 & 0,9886 & 0,9840 & 0,9794 & 0,9749 & 0,9705 & 0,9660 \\
& - & 1,4340 & 1,4320 & 1,4300 & 1,4290 & 1,4265 & 1,4250 & 1,4230 \\
\hline 0,7 & 1,0121 & 1,0076 & 1,0031 & 0,9986 & 0,9938 & 0,9893 & 0,9848 & 0,9803 \\
& - & 1,4350 & 1,4335 & 1,4320 & 1,4300 & 1,4280 & 1,4260 & 1,4245 \\
\hline 0,8 & 1,0251 & 1,0205 & 1,0159 & 1,0113 & 1,0065 & 1,0019 & 0,9973 & 0,9928 \\
& - & 1,4360 & 1,4350 & 1,4330 & 1,4310 & 1,4290 & 1,4270 & 1,4250 \\
\hline 0,9 & 1,0363 & 1,0316 & 1,0271 & 1,0225 & 1,0176 & 1,0130 & 1,0084 & 1,0038 \\
& - & 1,4375 & 1,4360 & 1,4340 & 1,4320 & 1,4300 & 1,4280 & 1,4260 \\
\hline 1,0 & 1,0468 & 1,0421 & 1,0374 & 1,0327 & 1,0280 & 1,0233 & 1,0187 & 1,0139 \\
& - & 1,4385 & 1,4370 & 1,4350 & 1,4330 & 1,4310 & 1,4290 & 1,4270 \\
\hline & & & & & & &
\end{tabular}

Tabla VI

Parámetros correspondientes a volúmenes molares $\left(\mathrm{cm}^{3} \cdot \mathrm{mol}^{-1}\right)$

\begin{tabular}{|c|c|c|c|c|c|c|c|c|c|}
\hline Miscela & & $278 \mathrm{~K}$ & $283 \mathrm{~K}$ & $288 \mathrm{~K}$ & $293 \mathrm{~K}$ & $298 \mathrm{~K}$ & $303 \mathrm{~K}$ & $308 \mathrm{~K}$ & $313 \mathrm{~K}$ \\
\hline $\begin{array}{l}\text { Triacetina } \\
\text { Butanol }\end{array}$ & $\bar{V}_{2}$ & $\begin{array}{r}185,8 \\
90,4\end{array}$ & $\begin{array}{r}186,7 \\
90,8\end{array}$ & $\begin{array}{r}187,6 \\
91,3\end{array}$ & $\begin{array}{r}188,5 \\
91,8\end{array}$ & $\begin{array}{r}189,3 \\
92,2\end{array}$ & $\begin{array}{r}190,3 \\
92,7\end{array}$ & $\begin{array}{r}191,2 \\
93,1\end{array}$ & $\begin{array}{r}192,1 \\
93,6\end{array}$ \\
\hline $\begin{array}{l}\text { Tributirina } \\
\text { Butanol }\end{array}$ & $\bar{V}_{2}$ & $\begin{array}{r}288,9 \\
90,2\end{array}$ & $\begin{array}{r}290,2 \\
90,6\end{array}$ & $\begin{array}{r}291,6 \\
91,1\end{array}$ & $\begin{array}{r}292,9 \\
91,6\end{array}$ & $\begin{array}{r}294,2 \\
92,0\end{array}$ & $\begin{array}{r}295,6 \\
92,5\end{array}$ & $\begin{array}{r}296,9 \\
92,9\end{array}$ & $\begin{array}{r}298,3 \\
93,4\end{array}$ \\
\hline $\begin{array}{l}\text { Tricaprilina } \\
\text { Butanol }\end{array}$ & $\bar{\nabla}_{2}$ & $\begin{array}{r}487,2 \\
90,2\end{array}$ & $\begin{array}{r}489,2 \\
90,6\end{array}$ & $\begin{array}{r}491,2 \\
91,1\end{array}$ & $\begin{array}{r}493,3 \\
91,6\end{array}$ & $\begin{array}{r}495,3 \\
92,0\end{array}$ & $\begin{array}{r}497,5 \\
92,4\end{array}$ & $\begin{array}{r}499,4 \\
92,9\end{array}$ & $\begin{array}{r}501,6 \\
93,4\end{array}$ \\
\hline $\begin{array}{l}\text { Tributirina } \\
\text { Etanol }\end{array}$ & $\overline{\mathrm{V}}_{2}$ & $\begin{array}{r}288,9 \\
57,2\end{array}$ & $\begin{array}{r}290,2 \\
57,5\end{array}$ & $\begin{array}{r}291,5 \\
57,7\end{array}$ & $\begin{array}{r}292,9 \\
58,0\end{array}$ & $\begin{array}{r}294,2 \\
58,4\end{array}$ & $\begin{array}{r}295,6 \\
58,7\end{array}$ & $\begin{array}{r}296,8 \\
58,9\end{array}$ & $\begin{array}{r}298,2 \\
59,3\end{array}$ \\
\hline $\begin{array}{l}\text { Tributirina } \\
\text { Hexanol }\end{array}$ & $\bar{V}_{2}$ & $\begin{array}{l}289,1 \\
123,4 \\
\end{array}$ & $\begin{array}{l}290,4 \\
123,9 \\
\end{array}$ & $\begin{array}{l}291,7 \\
124,5 \\
\end{array}$ & $\begin{array}{l}293,0 \\
125,1 \\
\end{array}$ & $\begin{array}{l}294,4 \\
125,7 \\
\end{array}$ & $\begin{array}{l}295,8 \\
126,3 \\
\end{array}$ & $\begin{array}{l}297,1 \\
126,8 \\
\end{array}$ & $\begin{array}{l}298,5 \\
127,4 \\
\end{array}$ \\
\hline \multicolumn{10}{|c|}{ Valores experimentales } \\
\hline $\begin{array}{l}\text { Etanol } \\
\text { Butanol } \\
\text { Hexanol } \\
\text { Triacetina } \\
\text { Tributirina } \\
\text { Tricaprilina }\end{array}$ & & $\begin{array}{r}57,4 \\
90,2 \\
123,1 \\
185,6 \\
288,8 \\
487,2\end{array}$ & $\begin{array}{r}57,7 \\
90,6 \\
123,7 \\
186,5 \\
290,1 \\
489,1\end{array}$ & $\begin{array}{r}58,0 \\
91,1 \\
124,2 \\
187,4 \\
291,5 \\
491,2\end{array}$ & $\begin{array}{r}58,3 \\
91,5 \\
124,8 \\
188,3 \\
292,8 \\
493,2\end{array}$ & $\begin{array}{r}58,7 \\
91,9 \\
125,4 \\
189,3 \\
294,1 \\
495,3\end{array}$ & $\begin{array}{r}59,0 \\
92,4 \\
125,9 \\
190,2 \\
295,5 \\
497,4\end{array}$ & $\begin{array}{r}59,3 \\
92,8 \\
126,5 \\
191,2 \\
296,8 \\
499,3\end{array}$ & $\begin{array}{r}59,7 \\
93,3 \\
127,1 \\
192,1 \\
298,2 \\
501,5\end{array}$ \\
\hline
\end{tabular}

En las miscelas triacetina-butanol y tributirina-hexanol, las pequeñas desviaciones del comportamiento ideal respecto al volumen molar, se deben a expansiones de volume atribuidas a una disminución del grado de estructuración de las miscelas (Mato, 1976), debido a la menor diferencia de tamaños entre las moléculas y a que disminuyen las asociaciones moleculares, produciéndose un aumento del espacio vacio

En el caso de las miscelas de tributirina y tricaprilina en butanol, la falta de semejanza en dimensiones entre 


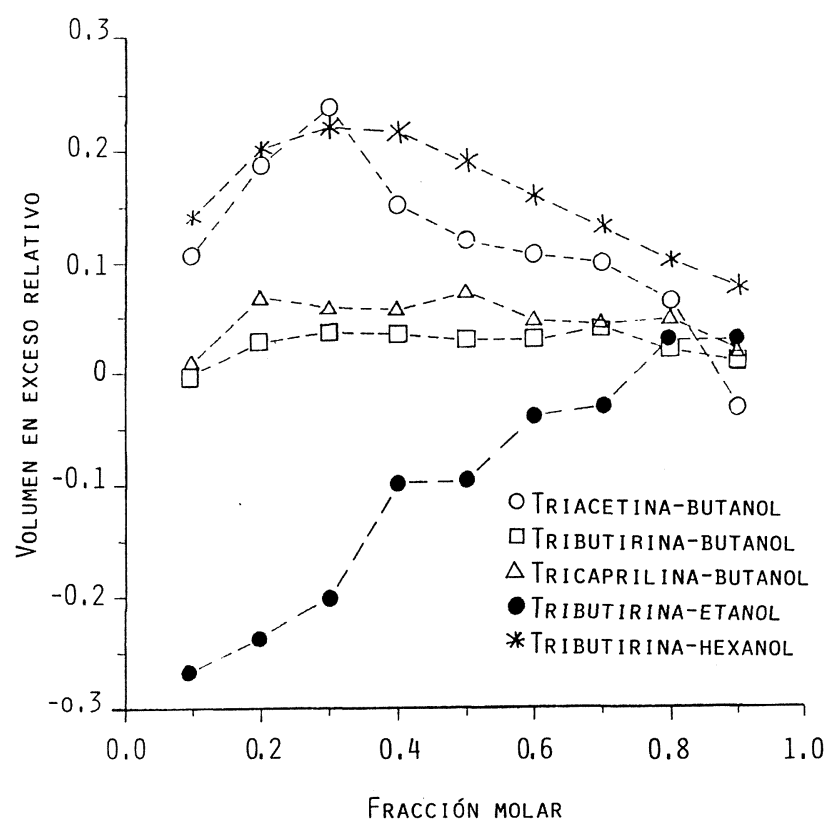

Figura 1

Variación del volumen molar en exceso relativo de las miscelas frente a la fracción molar de triglicérido a la temperatura de $303 \mathrm{~K}$.

las moléculas de los componentes (Flores, 1977a) y la menor intensidad entre los enlaces de hidrógeno en el butanol, justifica el comportamiento ideal de estas miscelas respecto a los volúmenes molares parciales.

Las contracciones y expansiones relativas de volumen molar no varían significativamente, en general, dentro del intervalo de temperatura estudiado para todos los sistemas.

\section{Relación volumen molar-temperatura}

Los valores de volumen molar de las miscelas expresados en centímetros cúbicos/mol, varian con la temperatura, expresada en kelvins según la ecuación:

$$
V=V_{293}+\alpha(T-293)
$$

siendo " $V_{293}$ " el valor del volumen molar a $293 \mathrm{~K}$ y " $(x$ " el coeficiente de dilatación térmico molar a presión constante, expresado en centímetros cúbicos/mol y Kelvin, para el intervalo de temperatura estudiado.

Los valores de los parámetros de ajuste de la regresión lineal de los volúmenes molares experimentales frente a "T-293", correspondientes a las miscelas estudiadas, se indican en la tabla (VII). En todos los casos los errores relativos para una probabilidad del $95 \%$ son inferiores a $0,02 \%$ y $2,1 \%$ respectivamente, resultando los valores de "VNJ" inferiores a 0,0168.

De los resultados reflejados en las tablas cabe destacar que los valores del parámetro " $\mathrm{V}_{293}$ " correspondientes a las miscelas estudiadas coinciden, dentro de su margen de error, con el valor experimental del volumen mo- lar a $293 \mathrm{~K}$ y que el valor del coeficiente de dilatación térmico molar a presión constante " $\alpha$ ", varía de la siguiente forma:

- Aumenta en todas las miscelas estudiadas a medida que lo hace la fracción molar del triglicérido en las mismas, debido a que los triglicéridos utilizados poseen un coeficiente de dilatación térmico molar a presión constante superior al de los alcoholes empleados.

- Para cada fracción molar de triglicérido, aumenta en las miscelas preparadas con butanol, desde triacetina a tricaprilina, en el mismo sentido en el que to hacen sus correspondientes coeficientes de dilatación térmico molar a presión constante.

- Para cada fracción molar de triglicérido, aumenta en las miscelas preparadas con tributirina, desde etanol a hexanol, en el mismo sentido en el que lo hacen sus correspondientes coeficientes de dilatación térmico molar a presión constante, aunque dichos aumentos son menos marcados que en el caso de los triglicéridos.

- En los cinco sistemas estudiados, se ha encontrado una relación lineal entre el parámetro " $\alpha$ " y la fracción molar de triglicérido " $x$ ", que puede expresarse de la forma:

$$
\alpha=\alpha_{1}^{A}+\left(\alpha_{2}^{\top}-\alpha_{1}^{A}\right) x
$$

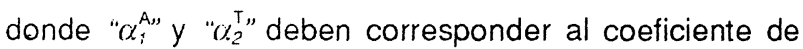
dilatación térmico molar a presión constante del alcohol y triglicérido, respectivamente, $y$ " $x$ " la fracción molar del triglicérido en la mezcla. Resultando los valores de "VNJ" inferiores a 0,3996 en todos los casos.

Los valores de los parámetros de ajuste de las regresiones lineales, expresados en centímetros cúbicos $/ \mathrm{mol}$ y Kelvin, junto con sus errores para una probabilidad del 95\% son:

\begin{tabular}{lcc}
\hline Miscela & $\alpha_{2}^{\top}$ & $\alpha_{1}^{\mathrm{A}}$ \\
\hline Triacetina-Butanol & $0,179 \pm 0,007$ & $0,092 \pm 0,002$ \\
Tributirina-Butanol & $0,269 \pm 0,004$ & $0,091 \pm 0,002$ \\
Tricaprilina-Butanol & $0,409 \pm 0,003$ & $0,090 \pm 0,002$ \\
Tributirina-Etanol & $0,265 \pm 0,007$ & $0,059 \pm 0,002$ \\
Tributirina-Hexanol & $0,269 \pm 0,004$ & $0,115 \pm 0,002$ \\
\hline
\end{tabular}

Los valores de " $\alpha$ " correspondientes a los productos de partida, expresados en centímetros cúbicos $/ \mathrm{mol}$ y Kelvin, junto con sus errores para una probabilidad del $95 \%$ son:

\begin{tabular}{lc}
\hline Componente & $\alpha\left(\mathrm{cm}^{3} \cdot \mathbf{m o l}^{-1} \cdot \mathbf{K}^{-1}\right)$ \\
\hline Triacetina & $0,185 \pm 0,002$ \\
Tributirina & $0,267 \pm 0,002$ \\
Tricaprilina & $0,409 \pm 0,004$ \\
Butanol & $0,089 \pm 0,001$ \\
Etanol & $0,066 \pm 0,001$ \\
Hexanol & $0,112 \pm 0,002$ \\
\hline
\end{tabular}

Del análisis de estos resultados se deduce que las miscelas de tributirina y tricaprilina en butanol cumplen la 
Tabla VII

Valores de los parámetros de ajuste " $V_{293}$ " $\left(\mathrm{cm}^{3} \cdot \mathrm{mol}^{-1}\right)$ y " $\alpha$ " $\left(\mathrm{cm}^{3} \cdot \mathrm{mol}^{-1} \cdot \mathrm{K}^{-1}\right)$

\begin{tabular}{|c|c|c|c|c|c|c|c|c|c|c|}
\hline & \multicolumn{2}{|c|}{$\begin{array}{c}\text { Triacetina } \\
\text { Butanol }\end{array}$} & \multicolumn{2}{|c|}{$\begin{array}{c}\text { Tributirina } \\
\text { Butanol }\end{array}$} & \multicolumn{2}{|c|}{$\begin{array}{c}\text { Tricaprilina } \\
\text { Butanol }\end{array}$} & \multicolumn{2}{|c|}{$\begin{array}{c}\text { Tributirina } \\
\text { Etanol }\end{array}$} & \multicolumn{2}{|c|}{$\begin{array}{c}\text { Tributirina } \\
\text { Hexanol }\end{array}$} \\
\hline & $V_{293}$ & $\alpha$ & $V_{293}$ & $\alpha$ & $V_{293}$ & $\alpha$ & $V_{293}$ & $\alpha$ & $V_{293}$ & $\alpha$ \\
\hline 0,0 & 91,52 & 0,089 & 91,52 & 0,089 & 91,52 & 0,089 & 58,34 & 0,066 & 124,79 & 0,112 \\
\hline 0,1 & 101,32 & 0,099 & 111,67 & 0,108 & 131,68 & 0,121 & 81,59 & 0,083 & 141,78 & 0,129 \\
\hline 0,2 & 111,10 & 0,110 & 131,85 & 0,126 & 171,91 & 0,154 & 105,03 & 0,101 & 158,69 & 0,145 \\
\hline 0,3 & 120,85 & 0,120 & 151,97 & 0,145 & 212,09 & 0,186 & 128,45 & 0,121 & 175,56 & 0,162 \\
\hline 0,4 & 130,50 & 0,128 & 172,11 & 0,163 & 252,27 & 0,219 & 151,98 & 0,141 & 192,38 & 0,178 \\
\hline 0,5 & 140,17 & 0,136 & 192,29 & 0,181 & 292,47 & 0,250 & 175,45 & 0,160 & 209,16 & 0,193 \\
\hline 0,6 & 149,85 & 0,145 & 212,39 & 0,198 & 332,67 & 0,282 & 198,95 & 0,182 & 225,93 & 0,208 \\
\hline 0,7 & 159,52 & 0,153 & 232,51 & 0,216 & 372,86 & 0,313 & 222,41 & 0,203 & 242,70 & 0,223 \\
\hline 0,8 & 169,15 & 0,163 & 252,68 & 0,232 & 412,96 & 0,346 & 245,98 & 0,225 & 259,44 & 0,238 \\
\hline 0,9 & 178,72 & 0,168 & 272,72 & 0,249 & 453,08 & 0,378 & 269,42 & 0,246 & 276,20 & 0,252 \\
\hline 1,0 & 188,37 & 0,185 & 292,82 & 0,267 & 493,24 & 0,409 & 292,82 & 0,267 & 292,82 & 0,267 \\
\hline
\end{tabular}

ley de aditividad respecto de los coeficientes de dilatación térmico molar a presión constante, ya que los valores de los parámetros " $\alpha_{2}^{\top \text { " }} y$ " $\alpha_{1}^{A}$ ", comprenden dentro de sus márgenes de error a los valores de $\alpha$ correspondientes a los productos de partida. En el caso de las miscelas de tributirina-etanol, triacetina-butanol y tributirina-hexanol los valores de " $\alpha$, no comprenden, dentro de los límites de error, los valores del coeficiente de dilatación térmico de estos alcoholes, en justa correspondencia con las contracciones y expansiones de volúmenes observadas experimentalmente para estos sistemas.

\subsection{Refracción molar de las miscelas}

Los valores de refracción molar de las miscelas se han determinado a partir de los valores experimentales del indice de refracción, de la densidad y del peso molecular medio mediante la expresión (Glastone, 1968):

Tabla VIII

Parámetros correspondientes a refracciones molares $\left(\mathrm{cm}^{3} \cdot \mathrm{mol}^{-1}\right)$

\begin{tabular}{lrrrrrrrr}
\hline Miscela & $\overline{\mathbf{R}}_{\mathbf{i}}$ & $\mathbf{2 8 3} \mathbf{K}$ & $\mathbf{2 8 8} \mathbf{K}$ & $\mathbf{2 9 3} \mathbf{K}$ & $\mathbf{2 9 8} \mathbf{K}$ & $\mathbf{3 0 3} \mathbf{K}$ & $\mathbf{3 0 8} \mathbf{K}$ & $\mathbf{3 1 3} \mathbf{K}$ \\
\hline Triacetina & $\overline{\mathrm{R}}_{2}$ & 48,6 & 48,7 & 48,7 & 48,7 & $\mathbf{4 8 , 7}$ & $\mathbf{4 8 , 8}$ & $\mathbf{4 8 , 8}$ \\
Butanol & $\overline{\mathrm{R}}_{1}$ & 22,1 & 22,1 & 22,1 & 22,1 & 22,2 & 22,2 & 22,2 \\
\hline Tributirina & $\overline{\mathrm{R}}_{2}$ & 76,3 & 76,3 & 76,3 & 76,4 & 76,5 & 76,5 & 76,6 \\
Butanol & $\overline{\mathrm{R}}_{1}$ & 22,1 & 22,1 & 22,1 & 22,1 & 22,2 & 22,2 & 22,2 \\
\hline Tricaprilina & $\bar{R}_{2}$ & 131,6 & 131,8 & 131,8 & 131,9 & 132,0 & 132,1 & 132,1 \\
Butanol & $\overline{\mathrm{R}}_{1}$ & 22,1 & 22,1 & 22,2 & 22,2 & 22,2 & 22,2 & 22,2 \\
\hline Tributirina & $\overline{\mathrm{R}}_{2}$ & 76,3 & 76,3 & 76,4 & 76,5 & 76,6 & 76,6 & 76,7 \\
Etanol & $\overline{\mathrm{R}}_{1}$ & 12,9 & 12,9 & 12,9 & 13,0 & 13,0 & 13,0 & 13,0 \\
\hline Tributirina & $\overline{\mathrm{R}}_{2}$ & 76,2 & 76,4 & 76,4 & 76,5 & 76,5 & 76,5 & 76,6 \\
Hexanol & $\overline{\mathrm{R}}_{1}$ & 31,4 & 31,4 & 31,4 & 31,4 & 31,4 & 31,5 & 31,5 \\
\hline Valores experimentales & & & & & & \\
\hline Etanol & & 12,9 & 12,9 & 12,9 & 12,9 & 12,9 & 12,9 & 12,9 \\
Butanol & & 22,1 & 22,1 & 22,1 & 22,1 & 22,2 & 22,2 & 22,2 \\
Hexanol & 31,4 & 31,4 & 31,4 & 31,4 & 31,5 & 31,5 & 31,5 \\
Triacetina & & 48,6 & 48,7 & 48,7 & 48,7 & 48,8 & 48,8 & 48,8 \\
Tributirina & & 76,2 & 76,4 & 76,4 & 76,4 & 76,5 & 76,5 & 76,6 \\
Tricaprilina & & 131,6 & 131,7 & 131,7 & 131,9 & 132,0 & 132,1 & 132,2 \\
\hline
\end{tabular}




$$
R=\frac{n^{2}-1}{n^{2}+2} \frac{M}{P}
$$

siendo " $R$ " la refracción molar de la miscela expresada en centímetros cúbicos/mol, " $P$ " la densidad, " $M$ " el peso molecular de la miscela y " $n$ " el índice de refracción.

\section{Relación refracción molar-composición}

De forma similar a como se ha procedido para el volumen molar de una mezcla binaria se llega para la refracción molar a una relación lineal entre la refracción molar experimental, " $R$ ", de la miscela y la fracción molar, " $x$ ", del triglicérido:

$$
R=\bar{R}_{1}+\left(\bar{R}_{2}-\bar{R}_{1}\right) x
$$

donde " $\bar{R}_{1}$ " y " $\bar{R}_{2}$ " son los valores de la refracción molar parcial del alcohol y del triglicérido respectivamente, que deben corresponder a los valores de la refracción molar parcial de dichos productos determinados experimentalmente, caso de que su comportamiento sea ideal. En la tabla VIII se recogen los valores de los parámetros " $\bar{R}_{1}$ " y " $\bar{R}_{2}$ " correspondientes a cada una de las miscelas estudiadas, expresados en centímetros cúbicos/mol y los valores experimentales de las refracciones molares de los alcoholes y triglicéridos utilizados, cuyo error se estima en $0,1 \mathrm{~cm}^{3} \cdot \mathrm{mol}^{-1}$ para una probabilidad del $95 \%$, resultando los valores de "VNJ" inferiores a 0,0023.

Los errores estimados en los valores de los parámetros de ajuste " $\bar{R}_{1}$ " y " $\bar{R}_{2}$ " para una probabilidad del $95 \%$, expresados en centímetros cúbicos/mol son:

\begin{tabular}{lll}
\hline \multicolumn{1}{c}{ Miscela } & $\overline{\boldsymbol{R}}_{\mathbf{2}}$ & $\overline{\boldsymbol{R}}_{\boldsymbol{1}}$ \\
\hline Triacetina-Butanol & $\pm 0,1$ & $\pm 0,1$ \\
Tributirina-Butanol & $\pm 0,2$ & $\pm 0,1$ \\
Tricaprilina-Butanol & $\pm 0,1$ & $\pm 0,1$ \\
Tributirina-Etanol & $\pm 0,2$ & $\pm 0,1$ \\
Tributirina-Hexanol & $\pm 0,2$ & $\pm 0,1$ \\
\hline
\end{tabular}

Del análisis de estos valores se deduce que el comportamiento de las miscelas estudiadas no difiere del correspondiente a la hipótesis de mezcla ideal, en relación a sus refracciones molares parciales. Esto conduce a pensar que las pequeñas contracciones de volumen observadas en el sistema tributirina-etanol y las expansiones de volumen observadas en los sistemas triacetinabutanol y tributirina-hexanol, no ocasionan alteración alguna significativa en las estructuras electrónicas de las moléculas constituyentes de las mezclas (Hirschfelder, 1969).

\section{Relación refracción molar-temperatura}

Dentro del intervalo de temperatura estudiado, la refracción molar de las mezclas permanece prácticamente constante. Este hecho está de acuerdo con la ecuación (5) que fue establecida como independiente de la temperatura o presión.

\section{BIBLIOGRAFIA}

Artículos de revista:

Flores Luque, V.: Gómez Herrera, C.: Tabernero de la Linde, P. (1974).- "Estudios físico-químicos de miscelas de aceites vegetales. I. Volúmenes parciales molares, indices de refracción y viscosidades de disoluciones de ácido láurico en hexano y ciclohexano".- Grasas y Aceites, 26, 216-220

Flores Luque, V.; Gómez Herrera. C.; Galán Vallejo, M. (1977a).- "Estudios físicoquimicos de miscelas de aceites vegetales. II. Volúmenes parciales molares, indices de refracción y viscosidades de disoluciones de ácido oleico en hexano y ciclohexano".- Grasas y Aceites 28, 77-83.

Flores Luque, V.; Gómez Herrera, C.; Plaza Delgado, A. (1977b).- "Estudios físico-químicos de miscelas de aceites vegetales. III. Tensiones superficiales en disoluciones de los ácidos láurico y oleico en hexano y ciclohexano. Valores de la función combinada (cociente tensión superficial/viscosidad cinemática)".--Grasas y Aceites 28, 337-344.

Flores Luque, V.; Gómez Herrera, C.; Cabrera Martín, J. (1977c).- "Estudios fisico-químicos de miscelas de aceites vegetales. IV. Relaciones entre tensión superficial y viscosidad cinemática en miscelas de aceites de oliva, ácido oleico o ácido láurico, con hexano o ciclohexano".- Grasas y Aceites 28, 415-419.

Flores Luque, V.; Galán Vallejo, M.; Cantero Moreno, D.; Quiroga Alonso J.M.; Gómez Herrera, C.; Cabrera Martín, J. (1979a).- "Estudios físico-quimicos de miscelas de aceites vegetales. VI. Volúmenes molares parciales, indices de refracción y viscosidades de disoluciones de los ácidos láurico u oleico en tricloroetileno o tetracloroetileno. Aplicación de la ecuación de fluidez de Hildebrand".- Grasas y Aceites 30, 75-81.

Flores Luque, V.; Galàn Vallejo, M.; Sales Márquez, D.; Gómez Herrera C. (1979b).- "Estudios físico-quimicos de miscelas de aceites vegetales. VII. Tensiones superficiales en disoluciones de los ácidos láurico u oleico en tricloroetileno o tetracloroetileno. Valores de la función combinada (cociente tensión superficial/viscosidad cinemática)".Grasas y Aceites 30, 355-359.

Flores Luque, V.: Gómez Herrera, C.: Galán Vallejo, M.: Sancho González, A.M. ${ }^{a}$ (1983).- "Estudios físico-químicos de miscelas de aceites vegetales. VIII. Volumen molar, refracción molar y viscosidad de las disoluciones de laurato de metilo en hexano, ciclohexano, tricloroetileno o tetracloroetileno".- Grasas y Aceites 34, 95-100.

Flores Luque, V.; Galán Vallejo, M.; López Torres, M.A.; Gómez Herrera C. (1984).- "Estudios físico-quimicos de miscelas de aceites vegetales. IX. Volumen molar, refracción molar y viscosidad de las disoluciones de laurato de n-hexilo en hexano, ciclohexano, tricloroetileno o tetracloroetileno".- Grasas y Aceites 35, 81-88.

Flores Luque, V.; Gómez Herrera, C.; Galán Vallejo, M.; López Torres, M.A. (1987).- "Estudios fisico-químicos de miscelas de aceites vegetales. XI. Refracción molar, volumen molar y viscosidad dinámica en disoluciones de aceites refinados de soja o de girasol en tricloroetileno o percloroetileno".- Grasas y Aceites 38, 104-109.

Jiménez, E.: Paz Andrade. M.I. (1974).- "Volúmenes de exceso de mezclas liquidas polares".- Anales de Química 70, 103-106.

Mato, F.; Fernández-Polanco, F. (1976).- "Propiedades dieléctricas de mezclas liquidas".- Anales de Química 72, 280-284

Muñoz Cueto, M. J.; Galán Vallejo, M.; Flores Luque, V. (1990a).- "Estudios fisico-quimicos de miscelas de aceites vegetales. XII. Volumen molar, refracción molar y viscosidad de disoluciones de miristato de metilo en ciclohexano, hexano, tetracloroetileno o tricloroetileno".Grasas y Aceites 41, 59-68.

Muñoz Cueto, M. ${ }^{a}$ J.; Galán Vallejo, M.; Martinez Moreno, J.M.; Flores Luque, V. (1990b).- "Estudios físico-químicos de miscelas de aceites vegetales. XIII. Volumen molar, refracción molar y viscosidad de disoluciones de palmitato de metilo en ciclohexano, hexano, tetracloroetileno o tricloroetileno".- Grasas y Aceites 41, 324-331.

Muñoz Cueto, $M \_J$ : Galán Vallejo, M.: Flores Luque, V. (1991) - "Estudios fisico-quimicos de miscelas de aceites vegetales. XIV. Volumen molar, refracción molar y viscosidad de disoluciones de estearato de metilo en ciclohexano, hexano, tetracloroetileno o tricloroetileno".Grasas y Aceites 42, 14-21.

Libros:

Glastone, S. (1968) - "Tratado de Quimica Fisica".- Ed. Aguilar, Madrid. Hirschfelder, J.O.; Curtis, Ch. F. and Bird, R.B. (1969).- "Molecular Theory of Gases and Liquids".- John Wiley and Sons. New York.

(Recibido: Marzo 1992) 